Acta Cryst. (1974). B30, 1570

\title{
Avicennin: Hypersymmetry in Space Group $\boldsymbol{P} \overline{\mathbf{1}}$ *
}

\author{
By Trng Fong LaI $\dagger$ and Richard E. Marsh \\ Arthur Amos Noyes Laboratory of Chemical Physics, California Institute of Technology, \\ Pasadena, California 91109, U.S.A.
}

(Received 30 January 1974; accepted 21 February 1974)

\begin{abstract}
Triclinic, $P \overline{1}, a=11.209$ (1), $b=10.196$ (2), $c=9.634$ (2) $\AA, \alpha=82.76$ (2), $\beta=109.43$ (1), $\gamma=123.61$ (1) , $Z=2 ; \mathrm{C}_{20} \mathrm{H}_{20} \mathrm{O}_{4}, \mathrm{~F}$. W. $324.38, D_{m}=1.25, D_{x}=1.249 \mathrm{~g} \mathrm{~cm}^{-3}$. The molecular structure is found to be based on a coumarin system fused at the 5,6 positions to an $\alpha$-pyran ring. The structure was solved by Patterson methods; failure of direct phasing methods can be traced to an 'aberrant phase relationship' due to the hypersymmetry of the molecule, as discussed by Thiessen \& Busing [Abs. Amer. Cryst. Assoc. (1973). Storrs, Connecticut, paper N-12, p. 183].
\end{abstract}

\section{Introduction}

Avicennin, $\mathrm{C}_{20} \mathrm{H}_{20} \mathrm{O}_{4}$, was isolated from the Rutaceae by Arthur \& Lee (1960) and also by Chow, Duffield \& Jefferies (1966). Three alternative structural formulae have been suggested on the basis of chemical and spectroscopic evidence (Arthur \& Lee, 1960; Arthur \& Ollis, 1963).<smiles>[R]c1c2c(c(O)c3ccc(=O)oc13)C=CC([M])(C)O2</smiles>

(I)<smiles>[R]c1c2c(c3oc(=O)ccc3c1OC)C=CC([M])(C)O2</smiles>

(II)

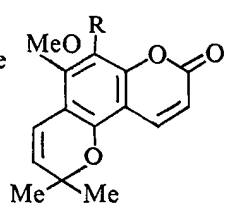

(III)

$$
\mathrm{R}=-\mathrm{CH}=\mathrm{CH}-\mathrm{CMe}=\mathrm{CH}_{2}
$$

We have now established that (III) represents the correct structure for avicennin. A preliminary account of this work was given by Lai \& Marsh (1969).

\section{Experimental}

Crystals of avicennin in the form of small yellow prisms were kindly supplied by $\mathrm{Mr} \mathrm{H}$. R. Arthur. Preliminary oscillation and Weissenberg photographs showed the crystals to be triclinic. Values of cell dimensions were obtained from a least-squares fit to measurements made on $0 k l, h 0 l$ and $h k 0$ Weissenberg photographs prepared in a Straumanis-type camera. The cell parameters and their standard deviations are: $a=11 \cdot 209(1), b=10 \cdot 196(2), c=9 \cdot 634(2) \AA, \alpha=82 \cdot 76(2)$,

* Contribution No. 4820 from the Arthur Amos Noyes Laboratory of Chemical Physics. This investigation was supported in part by Public Health Service Research Grant No. GM 16966, from the National Institute of General Medical Sciences, National Institutes of Health.

$\dagger$ Present address: Department of Chemistry, University of Hong Kong, Hong Kong. $\beta=109 \cdot 43(1), \gamma=123 \cdot 61(1)^{\circ}$. [The reduced cell has base vectors along [010], [110], and [001] and has dimensions $a^{\prime}=10.196, b^{\prime}=10.153, c^{\prime}=9.634 \AA, \alpha=103.93, \beta=$ $97 \cdot 24, \gamma=113 \cdot 15^{\circ}$. Our results are based on the nonreduced cell, which corresponds more closely to the crystal morphology.] The density measured by flotation is $1.25 \mathrm{~g} \mathrm{~cm}^{-3}$, and accordingly there are two molecules in the cell $\left(D_{x}=1.249 \mathrm{~g} \mathrm{~cm}^{-3}\right)$. The space group was preliminarily assumed to be $P \overline{1}$, and was confirmed by the successful refinement of the structure.

Intensity data were collected on a Datex-automated General Electric XRD-5 diffractometer using nickelfiltered $\mathrm{Cu} K \alpha$ radiation and a scintillation counter. All reflections within one hemisphere of reciprocal space out to $2 \theta=154^{\circ}$ were collected using a $\theta-2 \theta$ scan; background counts were collected at each scan extremum. A check reflection was monitored every 15 reflections. It indicated significant decay of the crystal; when the intensity had dropped to about $95 \%$ of the starting value, a second crystal was mounted to complete the data collection. The first crystal was an approximate cube with dimensions $0 \cdot 1 \times 0 \cdot 1 \times 0 \cdot 1$ $\mathrm{mm}$, and the second a square prism of dimensions $0.1 \times 0.15 \times 0.15 \mathrm{~mm}$. The scale factor between the two data sets and a crystal-decay factor were obtained from the measurements of the check reflection, and applied to all the data; absorption was assumed to be negligible $(\mu r \leq 0 \cdot 1)$. Of the 3649 reflections measured, 1243 had intensities less than one e.s.d. above background and were assigned weights of zero; observational variances $\sigma^{2}(I)$ for the other reflections were based on counting statistics for scan and background plus an additional term $(0.02 S)^{2}$, where $S$ is the scan count. Intensities and the standard deviations were then corrected for Lorentz and polarization factors and placed on an absolute scale by Wilson's (1942) method.

The hypersymmetry of the molecule and our ignorance of its geometry (see Introduction) led to rather severe problems in deriving the structure. We were unable to attain a correct solution by direct methods; eventually, Patterson methods were successful. 
Essentially all of the features of a sharpened Patterson map lay in two parallel sections, lying close to $(0 \overline{2} 2)$; the section passing through the origin (and which contains most of the intramolecular vectors) is shown in Fig. 1. While these sections clearly indicate that the molecules are approximately planar and that

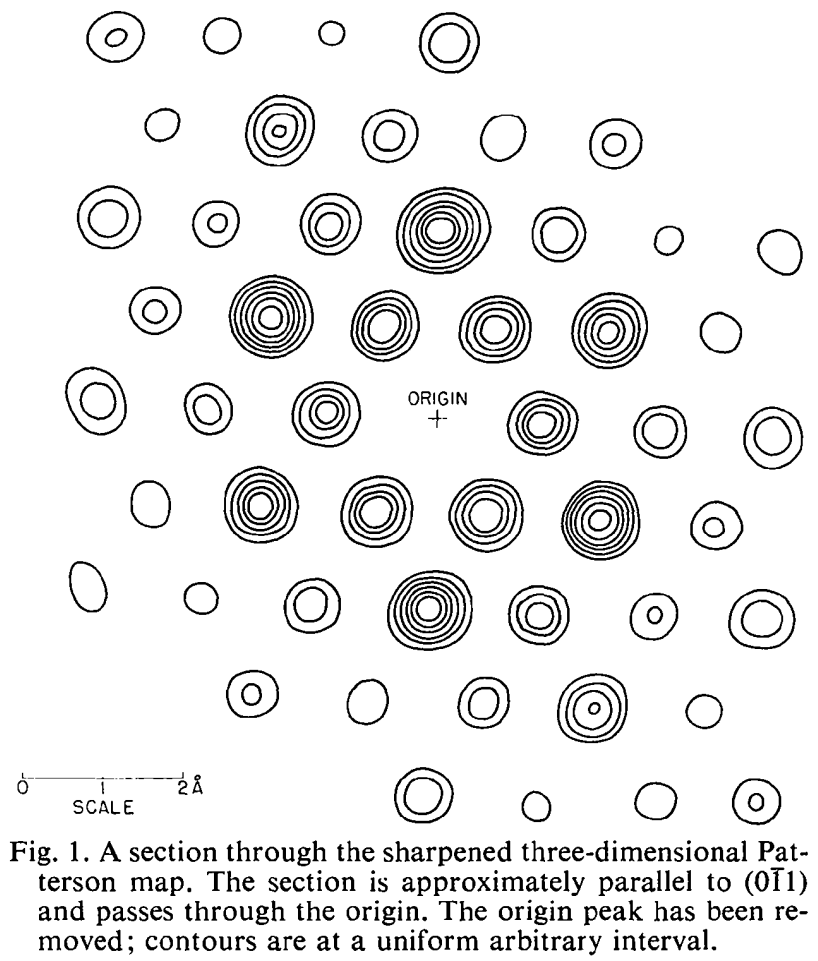

they lie midway between the $(0 \overline{2} 2)$ planes (packing considerations immediately rule out the possibility that they lie in planes containing centers of symmetry), they were of little help in establishing the geometry of the ring fusions or the positions of the side chains. Eventually we concluded, from the relative heights of the intramolecular vectors (Fig. 1), that a 16-atom molecular skeleton corresponding to structure (II) or (III) was more probable than one corresponding to structure (I). A large number of models apparently consistent with the Patterson section containing the intermolecular vectors werc tested, and a promising one was finally found. After two structure-factor, Fouriermap calculations, the remaining eight heavy atoms were recovered and the oxygen atoms were correctly identified. The $R$ index was $0 \cdot 50$.

After several cycles of least-squares adjustment of the coordinates and anisotropic temperature factors of the $\mathrm{C}$ and $\mathrm{O}$ atoms, the $R$ index $\left(=\sum|\Delta F| / \sum\left|F_{\text {obs }}\right|\right)$ was $0 \cdot 16$. A difference map then indicated the approximate positions of the hydrogen atoms. In the final refinement cycles, a list of 277 parameters was adjusted: coordinates for all 44 atoms, anisotropic temperature factors for the 24 heavy atoms, and a scale factor. The coordinates were included in one complete matrix, and the remaining parameters in a second. The isotropic temperature coefficients of the hydrogen atoms had previously been adjusted, to convergence, in a separate full-matrix refinement based on 1056 reflections with $\sin ^{2} \theta / \lambda^{2}$ less than $0 \cdot 2$.

During the last cycle no shift in the parameters was more than one third of its standard deviation. The final $R$ index for 2406 reflections of non-zero weight is $0 \cdot 113$ and the goodness of fit, $\left[\sum \omega\left(F_{o}^{2}-F_{c}^{2}\right)^{2} / m-s\right]^{1 / 2}$,

Table 1. The heavy-atom parameters and their standard deviations (in parentheses)

The coordinates have been multiplied by $10^{4}$. The anisotropic temperature factor is expressed in the form $\exp \left\{-\frac{1}{4}\left[h^{2} a^{* 2} B(11) \ldots\right.\right.$ $\left.\left.+2 k l b^{*} c^{*} B(23)\right]\right\}$; values of $B(i j)$ are multiplied by 10 .

$\begin{array}{lcccccccrr} & x & y & z & B(11) & B(22) & B(33) & B(12) & B(13) & B(23) \\ \mathrm{C}(1) & 4571(4) & 3361(5) & 1062(5) & 26(2) & 34(2) & 42(2) & 17(2) & 10(2) & -1(2) \\ \mathrm{C}(2) & 3178(5) & 2110(5) & 99(5) & 30(2) & 32(2) & 45(2) & 15(2) & 8(2) & -6(2) \\ \mathrm{C}(3) & 1846(5) & 1826(6) & 74(6) & 31(2) & 45(3) & 46(3) & 16(2) & 10(2) & -6(2) \\ \mathrm{C}(4) & 1796(5) & 2743(6) & 1043(6) & 29(2) & 47(3) & 50(3) & 19(2) & 10(2) & -2(2) \\ \mathrm{O}(5) & 3156(3) & 4000(3) & 1901(3) & 31(1) & 44(2) & 51(2) & 23(1) & 12(1) & -6(1) \\ \mathrm{C}(6) & 4535(5) & 4317(5) & 1937(5) & 30(2) & 38(2) & 44(3) & 23(2) & 13(2) & 3(2) \\ \mathrm{C}(7) & 5816(5) & 5604(5) & 2886(5) & 34(2) & 31(2) & 40(2) & 17(2) & 13(2) & 0(2) \\ \mathrm{C}(8) & 7185(5) & 5850(5) & 2924(5) & 30(2) & 30(2) & 46(3) & 12(2) & 8(2) & -6(2) \\ \mathrm{C}(9) & 7313(5) & 4934(5) & 2084(5) & 27(2) & 34(2) & 40(2) & 14(2) & 9(2) & -2(2) \\ \mathrm{C}(10) & 8745(5) & 5271(6) & 2049(6) & 25(2) & 42(2) & 56(3) & 13(2) & 12(2) & -7(2) \\ \mathrm{C}(11) & 8764(5) & 4242(6) & 1352(6) & 27(2) & 49(3) & 53(3) & 19(2) & 13(2) & -2(2) \\ \mathrm{C}(12) & 7341(4) & 2688(5) & 648(5) & 26(2) & 48(2) & 39(2) & 21(2) & 7(2) & -10(2) \\ \mathrm{O}(13) & 6009(3) & 2791(4) & 246(3) & 28(1) & 47(2) & 48(2) & 23(1) & 4(1) & -16(1) \\ \mathrm{C}(14) & 5990(5) & 3695(5) & 1161(5) & 35(2) & 37(2) & 43(2) & 24(2) & 16(2) & 4(2) \\ \mathrm{C}(15) & 5643(6) & 6543(5) & 3751(5) & 49(3) & 35(2) & 45(3) & 28(2) & 17(2) & 3(2) \\ \mathrm{O}(16) & 8527(3) & 7103(4) & 3800(4) & 33(1) & 45(2) & 53(2) & 16(1) & 6(1) & -16(1) \\ \mathrm{C}(17) & 9095(8) & 6684(9) & 5228(8) & 65(3) & 85(4) & 56(3) & 34(3) & -11(3) & -17(3) \\ \mathrm{C}(18) & 6680(6) & 7886(6) & 4563(7) & 53(3) & 46(3) & 66(3) & 26(2) & 22(2) & -6(2) \\ \mathrm{C}(19) & 6432(6) & 8737(6) & 5441(6) & 64(3) & 45(3) & 55(3) & 34(2) & 28(2) & 6(2) \\ \mathrm{C}(20) & 4836(8) & 8135(7) & 5307(8) & 75(3) & 49(3) & 62(3) & 39(3) & 28(3) & 4(2) \\ \mathrm{C}(21) & 7598(8) & 10001(8) & 6307(8) & 69(4) & 62(3) & 87(4) & 30(3) & 18(3) & -26(3) \\ \mathrm{C}(22) & 7137(6) & 1396(7) & 1677(7) & 38(2) & 49(3) & 63(3) & 23(2) & 10(2) & -1(2) \\ \mathrm{C}(23) & 7342(6) & 2312(9) & -840(7) & 38(2) & 92(4) & 53(3) & 34(3) & 13(2) & -23(3) \\ \mathrm{O}(24) & 669(3) & 2515(4) & 1203(4) & 33(1) & 73(2) & 73(2) & 28(2) & 17(2) & -12(2)\end{array}$


is 2-26. Calculations were carried out on an IBM-7094 computer under the CRYRM system (Duchamp, 1964). Atomic form factors for $C$ and $O$ were from International Tables for X-ray Crystallography (1962) and for H from Stewart, Davidson \& Simpson (1965).

The heavy-atom parameters and their standard deviations are given in Table 1; those of the hydrogen atoms, in Table 2 . Observed and calculated structure factors are listed in Table 3. The standard deviations in the parameters were calculated from the diagonal elements of the inverse matrices; since two separate matrices were collected, the e.s.d.'s may be a bit low.

Table 2. The hydrogen atom parameters and their standard deviations (in parentheses)

Values for the coordinates have been multiplied by $10^{3}$. The temperature factors are in the form $\exp \left(-B \sin ^{2} \theta / \lambda^{2}\right)$.

\begin{tabular}{lrrrr} 
& $x$ & $y$ & $z$ & \multicolumn{1}{c}{$\boldsymbol{B}$} \\
$\mathrm{H}(2)$ & $321(4)$ & $145(4)$ & $-48(4)$ & $1 \cdot 1(1 \cdot 0)$ \\
$\mathrm{H}(3)$ & $85(5)$ & $100(5)$ & $-65(5)$ & $3 \cdot 5(1 \cdot 2)$ \\
$\mathbf{H}(10)$ & $964(5)$ & $620(5)$ & $252(5)$ & $3 \cdot 5(1 \cdot 2)$ \\
$\mathrm{H}(11)$ & $972(5)$ & $444(5)$ & $137(5)$ & $2 \cdot 5(1 \cdot 1)$ \\
$\mathrm{H}(15)$ & $455(4)$ & $614(4)$ & $379(4)$ & $1 \cdot 0(1 \cdot 0)$ \\
$\mathrm{H}(17,1)$ & $1003(8)$ & $770(9)$ & $574(8)$ & $10 \cdot 6(2 \cdot 1)$ \\
$\mathrm{H}(17,2)$ & $833(8)$ & $640(9)$ & $578(8)$ & $11 \cdot 2(2 \cdot 2)$ \\
$\mathrm{H}(17,3)$ & $924(7)$ & $590(8)$ & $520(7)$ & $8 \cdot 8(1 \cdot 8)$ \\
$\mathrm{H}(18)$ & $780(5)$ & $832(6)$ & $462(5)$ & $4 \cdot 9(1 \cdot 4)$ \\
$\mathrm{H}(20,1)$ & $473(6)$ & $882(6)$ & $582(6)$ & $5 \cdot 4(1 \cdot 4)$ \\
$\mathrm{H}(20,2)$ & $418(6)$ & $801(6)$ & $424(7)$ & $5 \cdot 9(1 \cdot 5)$ \\
$\mathrm{H}(20,3)$ & $435(6)$ & $720(6)$ & $557(6)$ & $4 \cdot 5(1 \cdot 4)$ \\
$\mathrm{H}(21,1)$ & $875(7)$ & $1035(7)$ & $639(6)$ & $7 \cdot 4(1 \cdot 7)$ \\
$\mathrm{H}(21 \cdot 2)$ & $747(6)$ & $1054(7)$ & $697(6)$ & $7 \cdot 2(1 \cdot 6)$ \\
$\mathrm{H}(22 \cdot 1)$ & $711(6)$ & $161(6)$ & $269(6)$ & $5 \cdot 9(1 \cdot 5)$ \\
$\mathrm{H}(22,2)$ & $613(6)$ & $42(7)$ & $116(6)$ & $6 \cdot 0(1 \cdot 5)$ \\
$\mathrm{H}(22,3)$ & $805(5)$ & $130(5)$ & $187(5)$ & $3 \cdot 8(1 \cdot 3)$ \\
$\mathrm{H}(23,1)$ & $821(5)$ & $226(6)$ & $-60(5)$ & $4 \cdot 1(1 \cdot 3)$ \\
$\mathrm{H}(23,2)$ & $641(7)$ & $138(7)$ & $-125(6)$ & $6 \cdot 8(1 \cdot 6)$ \\
$\mathrm{H}(23,3)$ & $739(7)$ & $318(7)$ & $-165(7)$ & $7 \cdot 4(1 \cdot 7)$
\end{tabular}

A brief account of our unsuccessful attempts to derive the structure by direct phasing methods might be appropriate. Sayre (1952) relationships were obtained among the 481 reflections with $E$ values (Karle $\&$ Hauptman, 1956) greater than $1 \cdot 5$. Three signs were fixed to establish the origin, and two symbolic signs (Zachariasen, 1952) were introduced. Strongly consistent phases were obtained (by hand) for 297 reflections, and $E$ maps (Karle, Hauptman, Karle \& Wing, 1958) were calculated for each of the four combinations of symbolic phases. All of these maps featured extended sheets of peaks, but none of them suggested a reasonable structure. In retrospect, one of the $E$ maps (Fig. 2) contained a major portion of the structure, but severely masked by additional, very strong peaks.

During the phase expansion, an early entry into the list of established signs was the 444 reflection $(E=$ 2.68 ); its sign was established as positive, with 0.98 probability, on the basis of a $\sum_{1}$ relationship involving the 222 reflection $(E=3 \cdot 49)$. However, the final struc-

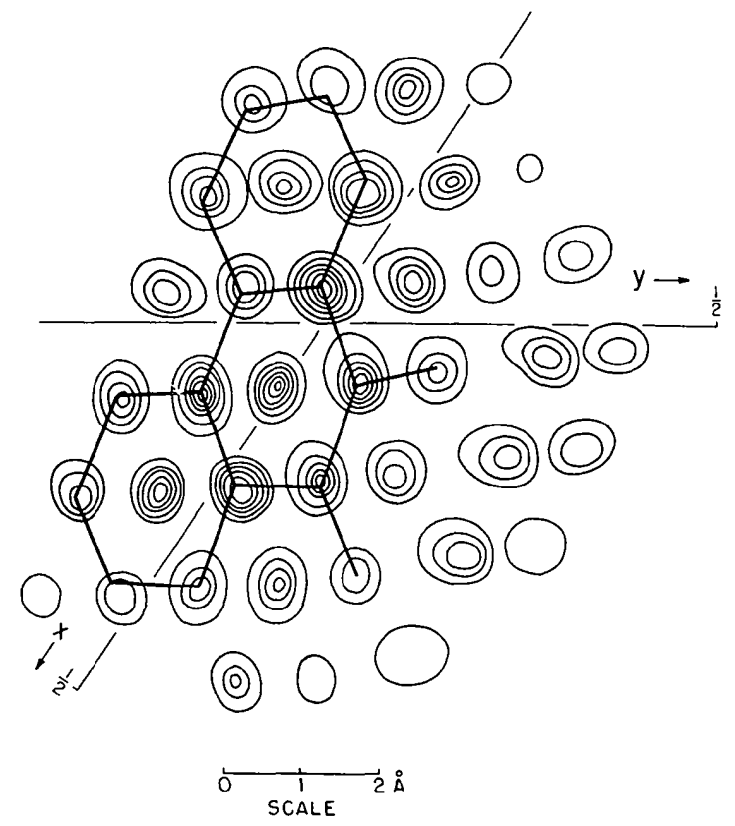

Fig. 2. A composite of a three-dimensional $E$ map, based on phases derived from an incorrect sign for the 444 reflection. The correct position of the ring system is indicated.
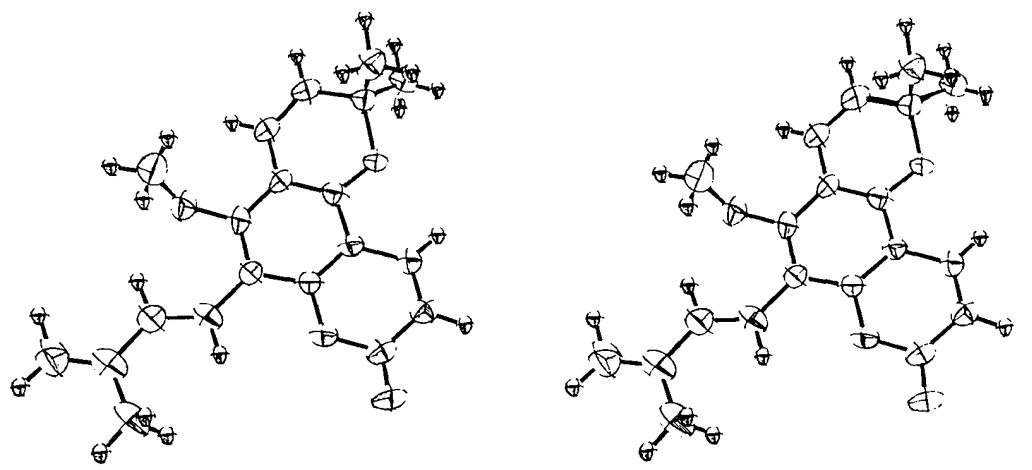

Fig. 3. A stereoscopic view of the molecule. Ellipsoids representing the thermal motions of the heavy atoms are drawn at the $50 \%$ probability level; the hydrogen atoms are represented as spheres of arbitrary radius. 
Table 3. Observed and calculated structure factors $(\times 10)$

A negative sign preceding $F$ (obs) indicates an intensity less than $1 \sigma$ above background; these reflections were given zero weights in the least-squares calculations. Reflections indicated with an asterisk were also given zero weight.

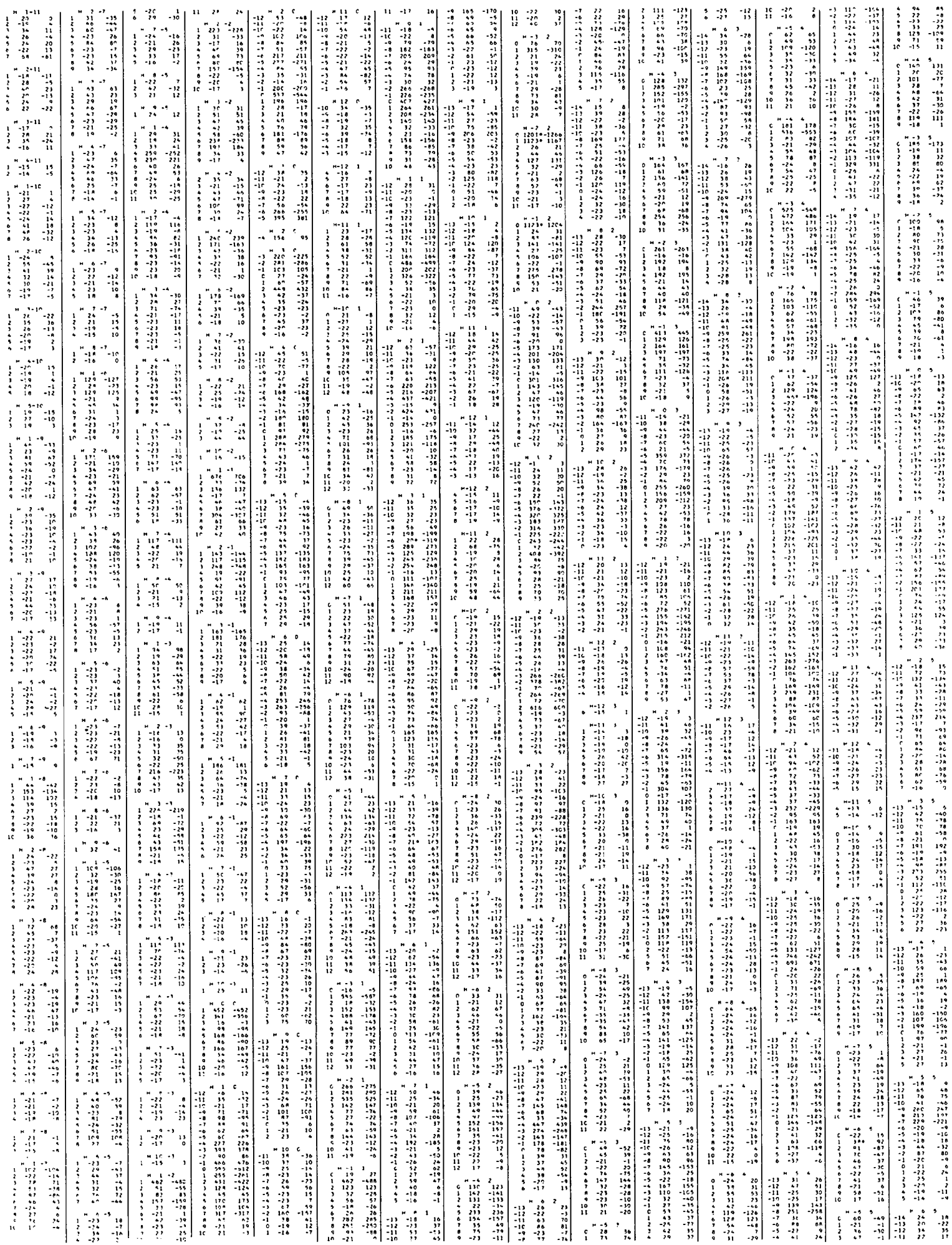


Table 3 (cont.)
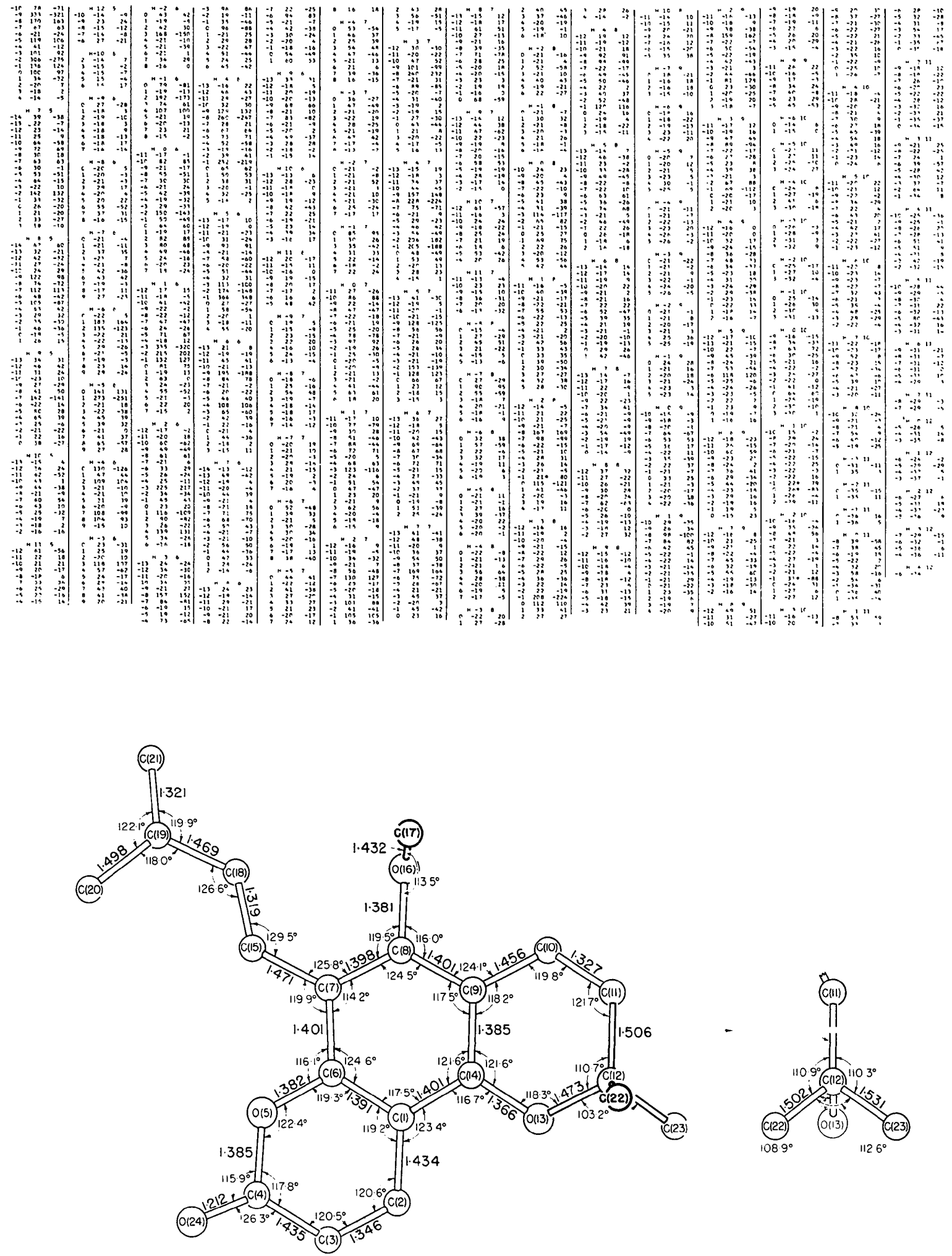

Fig. 4. Bond distances and angles involving the heavy atoms. Estimated standard deviations are in the range 0.007-0.011 $\AA$ for the distances and $0 \cdot 3-0 \cdot 5^{\circ}$ in the angles. 


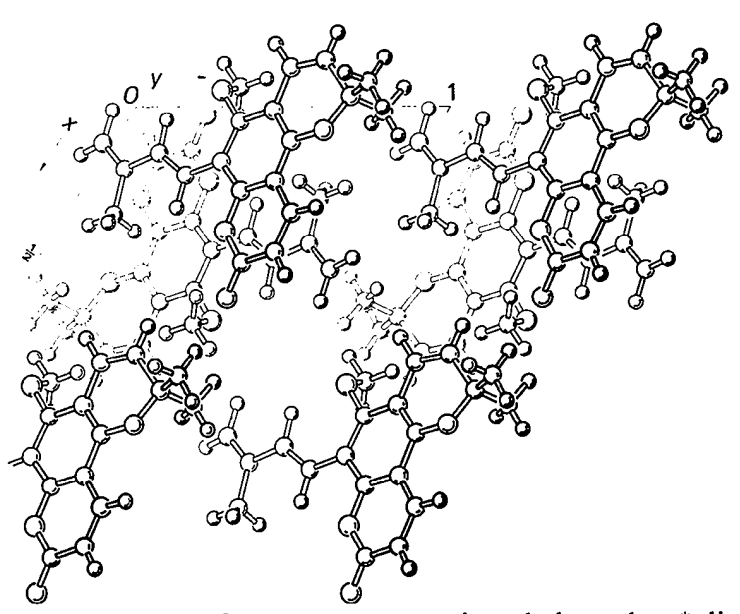

Fig. 5. A drawing of the structure, as viewed along the $c^{*}$ direction.

ture calls for the 444 reflection to have a negative sign. When, ex post facto, the sign of the 444 reflection was changed to negative and the sign expansion was repeated, the resulting $E$ map clearly indicated the correct structure. Dr David Duchamp (private communication) has since calculated for us the molecular transform of the planar ring system and has shown that, for the observed orientation of this system, the $\sum_{1}$ triplet involving the 222 and 444 reflections has an expected phase sum of $\pi$ rather than 0 . Thus, the difficulties that we experienced can be directly traced to the effect described by Thiessen \& Busing (1973).

\section{Results and discussion}

A stereoscopic drawing of the molecule is shown in Fig. 3; bond distances and angles involving the $\mathrm{C}$ and $\mathrm{O}$ atoms are given in Fig. 4. The observed values of the $20 \mathrm{C}-\mathrm{H}$ bond lengths range from 0.85 to $1.11 \AA$ with an average of $1.00 \pm 0.015 \AA$. The r.m.s. deviation of the individual values from this average is $0.066 \AA$, in essentially exact agreement with the estimated uncertainties in the coordinates of the hydrogen atoms (Table 2).

The molecule is significantly non-planar; deviations from the least-squares plane passed through the 10 ring atoms of the coumarin system are given in Table 4. The largest puckering occurs in the $\alpha$-pyran ring, where $C(12)$ is displaced by nearly $0.5 \AA$ from the coumarin plane. The relatively large displacement of $\mathrm{O}(24)$ is rather surprising; it quite clearly results from intermolecular forces involving the methyl groups $\mathrm{C}(22)$ and $C(23)$ of an adjacent molecule related by translation along the $a$ axis (see Fig. 5), the most important distances being $\mathrm{C}(23) \cdots \mathrm{O}(24), 3 \cdot 491 \AA$ and $\mathrm{H}(23,1) \cdots$ $\mathrm{O}(24), 2 \cdot 61 \AA$.
Table 4. Deviations of the heavy atoms from the best plane of the coumarin ring

The equation of the best plane, with the coefficients equal to direction cosines with respect to the crystallographic axes, is: $-0.1035 a x+0.6161 b y-0.7027 c z=0.861 \AA$. Atoms included in the calculation of the best plane are marked with an asterisk.

$\begin{array}{lrlr} & \text { Deviation } & & \text { Deviation } \\ \mathrm{C}(1)^{*} & 0.001 \AA & \mathrm{O}(13) & 0.029 \AA \\ \mathrm{C}(2)^{*} & 0.029 & \mathrm{C}(14)^{*} & -0.021 \\ \mathrm{C}(3)^{*} & 0.022 & \mathrm{C}(15) & 0.055 \\ \mathrm{C}(4)^{*} & -0.052 & \mathrm{O}(16) & 0.038 \\ \mathrm{O}(5)^{*} & -0.001 & \mathrm{C}(17) & -1.257 \\ \mathrm{C}(6)^{*} & 0.013 & \mathrm{C}(18) & 0.229 \\ \mathrm{C}(7)^{*} & 0.031 & \mathrm{C}(19) & 0.198 \\ \mathrm{C}(8)^{*} & 0.001 & \mathrm{C}(20) & 0.095 \\ \mathrm{C}(9)^{*} & -0.021 & \mathrm{C}(21) & 0.270 \\ \mathrm{C}(10) & 0.048 & \mathrm{C}(22) & -1.948 \\ \mathrm{C}(11) & -0.129 & \mathrm{C}(23) & 0.308 \\ \mathrm{C}(12) & -0.464 & \mathrm{O}(24) & -0.173\end{array}$

The most intimate stacking between pairs of molecules occurs across the center of symmetry at $\left(\frac{1}{2}, \frac{1}{2}, 0\right)$, the closest contact distances being $\mathrm{C}(2) \cdots \mathrm{C}(8) 3.405 \AA$; $\mathrm{C}(4)$...C(10) $3.461 \AA$; C(6) ...C(14) $3.463 \AA$; $O(5) \cdots$ $\mathrm{O}(13) 3.472 \AA$; and $\mathrm{C}(3) \cdots \mathrm{C}(9) 3.500 \AA$. Stacking across the center at $\left(\frac{1}{2}, \frac{1}{2}, \frac{1}{2}\right)$ is considerably less intimate; apart from a $\mathrm{C}(17) \cdots \mathrm{O}(24)$ distance of $3.525 \AA$, which can hardly represent a stacking interaction, the shortest contact is $\mathrm{C}(6) \cdots \mathrm{C}(20), 3.582 \AA$.

We are grateful to Dr David Duchamp for calculating the molecular transform, to Miss Lillian Casler for preparing most of the figures, and to Miss Allison Kimball for preparing the manuscript.

\section{References}

Arthur, H. R. \& Lee, C. M. (1960). J. Chem. Soc. p. 4654. Arthur, H. R. \& Ollis, W. D. (1963). J. Chem. Soc. p. 3910.

Chow, P. W., Duffield, A. M. \& Jefferies, P. R. (1966). Aust. J. Chem. 19, 483.

Duchamp, D. J. (1964). Abs. Amer. Cryst. Assoc., Bozeman, Montana, paper B-14, p. 29.

International Tables for X-ray Crystallography (1962). Vol. III, pp. 202-203. Birmingham: Kynoch Press.

Karle, I. L., Hauptman, H., Karle, J. \& Wing, A. B. (1958). Acta Cryst. 11, 257-263.

Karle, J. \& Hauptman, H. (1956). Acta Cryst. 9, 635-651.

LaI, T. F. \& Marsh, R. E. (1969). Abs. Amer. Cryst. Assoc., Seattle, Washington, paper D-2, p. 32.

SAYre, D. (1952). Acta Cryst. 5, 60-65.

SteWART, R. F., Davidson, E. R. \& Simpson, W. T. (1965). J. Chem. Phys. 42, 3175-3187.

Thiessen, W. E. \& Busing, W. R. (1973). Abs. Amer. Cryst. Assoc., Storrs, Connecticut, paper N-12, p. 183.

Wilson, A. J. C. (1942). Nature, Lond. 150, 151-152.

Zachariasen, W. H. (1952). Acta Cryst. 5, 68-73. 